\title{
Three-Dimensional Ultrasound Volume Measurements in an Aortic Aneurysm Model
}

Lydia Johnsen ${ }^{1,2}$, MSc. Torbjørn Jonung ${ }^{1,2}$, MD PhD. Elin Laxdal ${ }^{3}$, MD PhD. Gustav Pedersen ${ }^{1,2}$, MD PhD.

${ }^{1}$ Department of Vascular Surgery, Haukeland University Hospital, Bergen, Norway

2Department of Clinical Science, University of Bergen, Norway

${ }^{3}$ Department of Vascular Surgery, Landspitali, Reykjavik, Iceland

\section{Keywords}

Abdominal aortic aneurysm

Endovascular aneurysm repair

In vitero

Three-dimensional

Ultrasonography

\section{Abstract}

Measuring the aneurysm sac's size is vital in postoperative surveillance following endovascular treatment of aortic aneurysms. A three-dimensional ultrasound technique may enable accurate volume measurements. However, there is no validation of any commercially available electro-mechanical 3D ultrasound equipment or of the software used when measuring the volume of the aortic aneurysm sac. This investigation used a phantom model to study a three-dimensional ultrasound technique on aortic aneurysm sac volume measurements. High volume measurement accuracy indicates that this method may be useful for postoperative surveillance following endovascular aortic aneurysm operations. These results must be confirmed in clinical studies.

\section{Introduction}

Abdominal aortic aneurysm ( $A A A)$ is a potentionally life-threatening condition, and without repair it is fatal. Repair is recommended in aneurysm diameter $>5.5 \mathrm{~cm}^{1}$. Endovascular aneurysm repair (EVAR) of AAA has increased gradually since the early 1990s. Compared to traditional open surgery for AAA, EVAR is associated with less surgical trauma and faster recovery. Consequently, EVAR allows surgeons to treat patients with AAA who might otherwise not have been able to undergo surgery. The major disadvantages of EVAR treatment include leakage of blood into the aortic sac (endoleak, EL), graft migration, and increased risk of aortic sac rupture; thus, EVAR requires lifetime follow-up ${ }^{2}$. 
Contrast-enhanced computed tomographic angiography (CTA) is considered the gold standard for follow-up after EVAR. However, repeat CTA is harmful due to the accumulated radiation dosage and the association between arterial contrast dyes and reduced kidney function ${ }^{3}$.

Ultrasound may be used as an alternative method to CTA for follow-up after EVAR, as it is less harmful. However, conventional ultrasound has shown poor sensitivity in detecting $\mathrm{EL}^{4}$. Aneurysms can be monitored by measuring whether the size of the AAA sac has changed. It is only necessary to treat ELs that have caused an increase in the size of the AAA sac ${ }^{5-6}$.

The size of the AAA sac is usually determined by measuring its diameter. Three-dimensional ultrasound (3D US) volume measurements using a modified research probe and software systems have shown to be more precise and have better accuracy than diameter measurements when detecting changes in various organs ${ }^{7-10}$. However, there is no validation of any commercially available electro-mechanical 3D ultrasound equipment or of the software used when measuring the volume of the aortic aneurysm sac. The aim of this study was to validate a commercially available 3D US method for ultrasound volume measurements in an AAA model.

\section{Methods}

The study design was experimental. We used balloons filled with water as phantom aneurysm sacs for the 3D US volume measurements $(n=30)^{11-13}$. After immersing the waterfilled balloons in a bucket of water, we placed an ultrasound probe at the surface of the water. The phantom aneurysm in the water-filled bucket and the water surface simulated the distance between an AAA and the skin surface (Fig. 1). Once the test was complete, we emptied the phantom aneurysm sacs into measuring cups to determine the true volumes.

The ultrasound equipment used for the experiment was Logiq 9 (GE Healthcare, Milwaukee, WI, USA). The ultrasound probe was a 3D/4D RAB 2-5D curved linear electromechanical wideband array transducer, 1-4 MHz frequencies (GE Healthcare). This probe consists of a conventional 2D transducer placed in a container (housing). Inside the container, the 2D probe sweeps back and forth, creating a 3D image. When starting to record 3D volume, the probe swept over the phantom aneurysm to collect data (Figs. 2 and 3 ). The sweep range was maximum $70^{\circ}$, and the sweep time for volume recording was approximately 3 seconds.

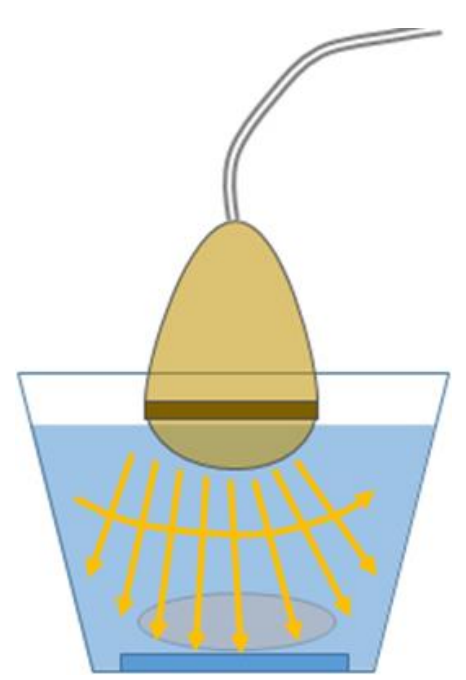

Figure 1: Water-filled balloon simulating an abdominal aortic aneurysm attached to the bottom of a water-filled bucket. The threedimensional ultrasound probe was placed into water at a specific distance from the phantom, simulating the distance between skin and abdominal aortic aneurysm. Arrows show the ultrasound sweep. 


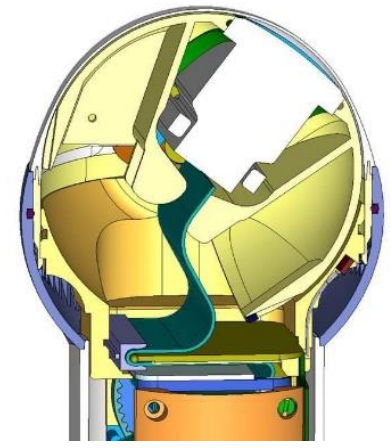

Figure 2: Sectional view of the RAB2-5D probe, demonstrating the included two-dimensional probe. (Permission to include this figure obtained from GE Ultrasound.)

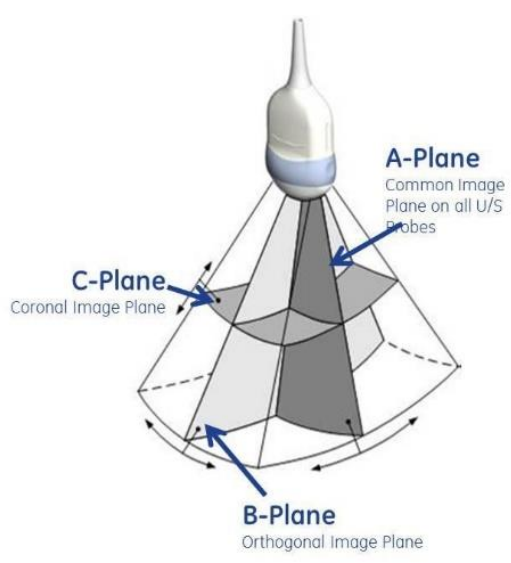

Figure 3: Three-dimensional scanning planes in threedimensional ultrasound. (Permission to include this figure obtained from GE Ultrasound.)

We used VOCAL ${ }^{\text {TM }}$ (Virtual Organ Computer-Aided Analysis; General Electric Medical Systems, Kretztechnik, Zipf, Austria) to calculate the volume of the aneurysm, with the function "volume rendering" (volume calculation). The recorded volume was displayed in six different planes and then outlined manually. The VOCAL ${ }^{\mathrm{TM}}$ program reconstructed a 3D figure; the volume of the aneurysm model is shown in $\mathrm{cm}^{3}$ ( $\mathrm{ml}$ ) (Fig. 4).

The ultrasound procedure included postprocessing that took about 5-10 minutes for each phantom.

Two technicians independent of one another performed two separate sets of 3D US volume measurements. A third technician, unaware of the ultrasound measurements, emptied the balloons and measured the true volumes.

We analyzed the results with SPSS 15.0 (released 2006; SPSS Inc., Chicago, IL, USA). Correlations between true and estimated volumes were expressed by Pearson's correlation. We calculated mean percentage errors. Inter-rater agreement was expressed by a BlandAltman plot (limits of agreement). Bias was estimated as the average difference

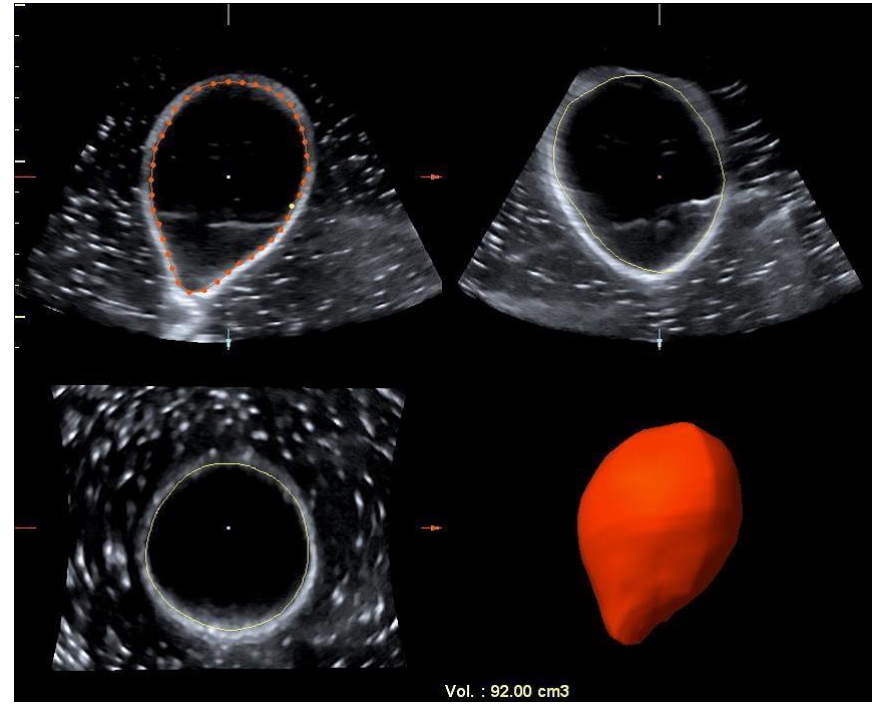

Figure 4: Volume measurement by VOCAL ${ }^{\mathrm{TM}}$ software. Upper left corner: A plane, original image. Red dots and lines show the manually outlined borders of the phantom. Upper right corner: B plane, reconstructed orthogonal image. Lower left corner: $C$ plane, reconstructed coronal image. Lower right corner: Three-dimensional model reconstruction based on the manually outlined borders in six different A planes. between estimated volumes and true volumes. A $P$ value of 0.05 was considered significant. 
Johnsen, Jonung, Laxdal, Pedersen

Three-dimensional Ultrasound Volume Measurements in an Aortic Aneurysm Model

\section{Results}

The true volumes of the water-filled balloons varied from $52 \mathrm{ml}$ to $242 \mathrm{ml}$. The average volume was $132.2 \mathrm{ml}$. Technician A estimated $129 \mathrm{ml}$ on average, and technician B estimated $124.4 \mathrm{ml}$ on average.

The estimated volumes as measured by 3D US deviated from the true volumes from $-15 \mathrm{ml}$ to $+6 \mathrm{ml}$ for investigator $\mathrm{A}$, and from $-18 \mathrm{ml}$ to $+15 \mathrm{ml}$ for investigator $\mathrm{B}$. Technician $\mathrm{A}$ underestimated the volume by $-3.2 \mathrm{ml}$ on average, while technician $B$ underestimated the volume by $-6.3 \mathrm{ml}$.

Inter-rater agreement between the technicians was very good, as measured by correlation: $r^{2}=0.98, r=0.99$ ( $p<0.0001$; Fig. 5).

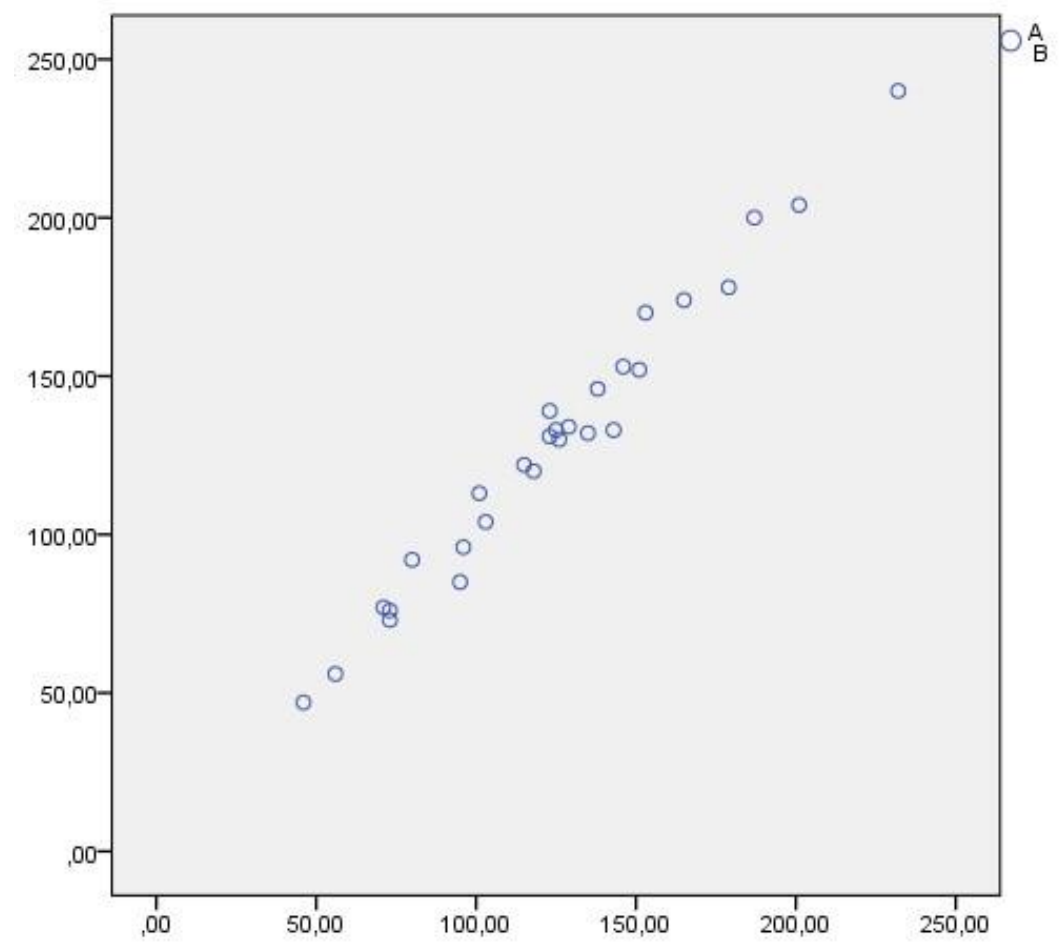

Figure 5: Inter-rater agreement. Two-tailed Pearson's correlation coefficient showing the correlation between volumes measured by technicians $A$ and $B$.

The correlation between technician A's estimated volume measurements and the true volumes was excellent: $r^{2}=0.98, r=0.99(p<0.0001)$, as was the correlation between technician $\mathrm{B}^{\prime} \mathrm{s}$ estimated volume measurements and the true volumes: $r^{2}=0.97, r=0.98(p<0.0001 ;$ Fig. 6$)$. 


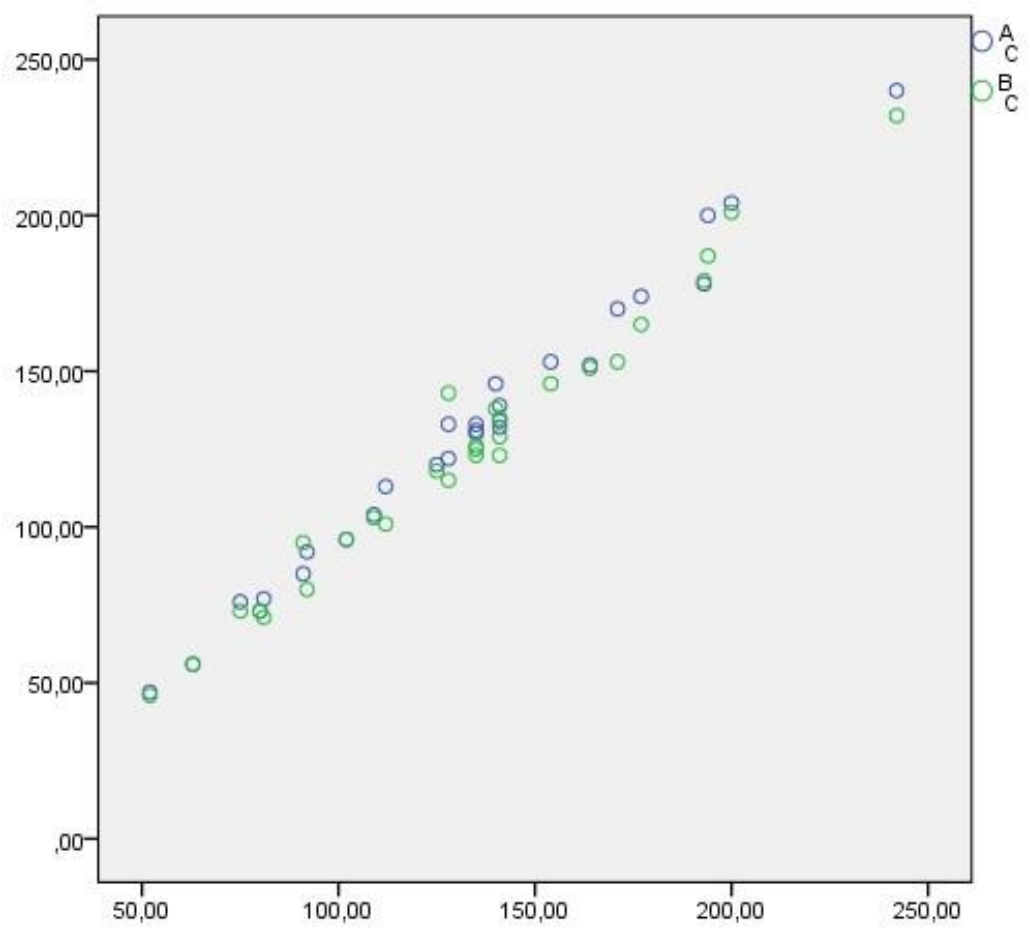

Figure 6: Correlation between true volumes (C) and experimental volumes measured by technicians $A$ and $B$ using three-dimensional ultrasound. Two-tailed Pearson's correlation coefficient.

According to percentage deviation calculations, deviations from the true volumes were $5.73 \%$ overall (4.02\% for technician A and $7.44 \%$ for technician B) (Table 1 ). Limits of agreement between the ultrasound technicians ranged from $-8.8 \mathrm{ml}$ to $+17.4 \mathrm{ml}$, with an average of $4.3 \mathrm{ml}$ (Bland-Altman plot, Fig. 7). Overall, 3D US accurately and precisely measured the volumes of the water-filled balloons in our phantom aneurysm model. 
Johnsen, Jonung, Laxdal, Pedersen

Three-dimensional Ultrasound Volume Measurements in an Aortic Aneurysm Model

\begin{tabular}{|c|c|c|c|c|c|c|c|c|c|c|}
\hline $\begin{array}{c}\text { Phan } \\
\text { tom } \\
\mathrm{nr}\end{array}$ & $\begin{array}{c}\text { Investi } \\
\text { gator A } \\
\text { ml }\end{array}$ & $\begin{array}{c}\text { Investi } \\
\text { gator } \\
\text { B ml }\end{array}$ & $\begin{array}{c}\text { True } \\
\text { volume } \\
\text { ml }\end{array}$ & $\begin{array}{c}\text { Diff } \\
\text { A/B ml }\end{array}$ & $\begin{array}{c}\text { Diff } \\
\text { A/C } \\
\mathrm{ml}\end{array}$ & $\begin{array}{l}\text { Diff } \\
\text { B/C ml }\end{array}$ & $\begin{array}{c}\text { Diff A/C } \\
\%\end{array}$ & $\begin{array}{c}\text { Diff } B / C \\
\%\end{array}$ & $A+B / 2$ & $\begin{array}{c}\text { Diff } \\
A+B / C \\
\%\end{array}$ \\
\hline 1 & 132 & 135 & 141 & -3 & -9 & -6 & $6,38 \%$ & $4,25 \%$ & 133,5 & $5,31 \%$ \\
\hline 2 & 122 & 115 & 128 & 7 & -6 & 13 & $4,68 \%$ & $10,15 \%$ & 118,5 & $7,41 \%$ \\
\hline 3 & 240 & 232 & 242 & 8 & -2 & -10 & $0,82 \%$ & $4,13 \%$ & 236 & $2,48 \%$ \\
\hline 4 & 113 & 101 & 112 & 12 & 1 & -11 & $0,89 \%$ & $9,82 \%$ & 107 & $5,36 \%$ \\
\hline 5 & 133 & 125 & 135 & 8 & -2 & -10 & $1,48 \%$ & $7,40 \%$ & 129 & $4,44 \%$ \\
\hline 6 & 130 & 126 & 135 & 4 & -5 & -9 & $3,70 \%$ & $6,66 \%$ & 128 & $5,18 \%$ \\
\hline 7 & 133 & 143 & 128 & -10 & 5 & 15 & $3,90 \%$ & $11,71 \%$ & 138 & $7,81 \%$ \\
\hline 8 & 96 & 96 & 102 & 0 & -6 & -6 & $5,88 \%$ & $5,88 \%$ & 96 & $5,88 \%$ \\
\hline 9 & 174 & 165 & 177 & 9 & -3 & -12 & $1,69 \%$ & $6,77 \%$ & 169,5 & $4,23 \%$ \\
\hline 10 & 73 & 73 & 80 & 0 & -7 & -7 & $8,75 \%$ & $8,75 \%$ & 73 & $8,75 \%$ \\
\hline 11 & 146 & 138 & 140 & 8 & 6 & -2 & $4,28 \%$ & $1,42 \%$ & 142 & $2,85 \%$ \\
\hline 12 & 131 & 123 & 135 & 8 & -3 & -12 & $2,22 \%$ & $8,88 \%$ & 127 & $5,55 \%$ \\
\hline 13 & 176 & & & & & & & & & \\
\hline 14 & 92 & 80 & 92 & 8 & 0 & -12 & $0 \%$ & $13,04 \%$ & 86 & $6,52 \%$ \\
\hline 15 & 104 & 103 & 109 & 1 & -5 & -6 & $4,58 \%$ & $5,50 \%$ & 103,5 & $5,04 \%$ \\
\hline 16 & 85 & 95 & 91 & -10 & -6 & 4 & $6,59 \%$ & $4,39 \%$ & 90 & $5,49 \%$ \\
\hline 17 & 76 & 73 & 75 & 3 & 1 & -2 & $1,33 \%$ & $2,66 \%$ & 74,5 & $1,99 \%$ \\
\hline 18 & 153 & 146 & 154 & 7 & -1 & -8 & $0,64 \%$ & $5,19 \%$ & 149,5 & $2,92 \%$ \\
\hline 19 & 120 & 118 & 125 & -2 & -5 & -7 & $4,00 \%$ & $5,60 \%$ & 119 & $4,80 \%$ \\
\hline 20 & 134 & 129 & 141 & 5 & -7 & -12 & $4,96 \%$ & $8,51 \%$ & 131,5 & $6,74 \%$ \\
\hline 21 & 200 & 187 & 194 & 13 & 6 & 7 & $3,09 \%$ & $3,60 \%$ & 193,5 & $3,35 \%$ \\
\hline 22 & 56 & 56 & 63 & 0 & -7 & -7 & $11,11 \%$ & $11,11 \%$ & 56 & $11,11 \%$ \\
\hline 23 & 152 & 151 & 164 & 1 & -12 & -13 & $7,31 \%$ & $7,92 \%$ & 151,5 & $7,62 \%$ \\
\hline 24 & 48 & 46 & 52 & 2 & -4 & -6 & $7,69 \%$ & $11,53 \%$ & 47 & $9,61 \%$ \\
\hline 25 & 188 & & & & & & & & & \\
\hline 26 & 170 & 153 & 171 & 17 & -1 & -18 & $0,58 \%$ & $10,52 \%$ & 161,5 & $5,55 \%$ \\
\hline 27 & 178 & 179 & 193 & -1 & -15 & -14 & $7,77 \%$ & $7,25 \%$ & 178,5 & $7,51 \%$ \\
\hline 28 & 204 & 201 & 200 & 3 & 4 & 1 & $2,00 \%$ & $0,50 \%$ & 202,5 & $1,25 \%$ \\
\hline 29 & 139 & 123 & 141 & 16 & -2 & -18 & $1,41 \%$ & $12,76 \%$ & 131 & $7,09 \%$ \\
\hline 30 & 77 & 71 & 81 & 6 & -4 & -10 & $4,93 \%$ & $12,34 \%$ & 74 & $8,64 \%$ \\
\hline Mean & 132,50 & 124,39 & 132,18 & 4,29 & $-3,18$ & $-6,36$ & $4,02 \%$ & $7,44 \%$ & 126,68 & $5,73 \%$ \\
\hline $\begin{array}{c}\text { Max } \\
\text { value }\end{array}$ & 240,00 & 232,00 & 242,00 & 17,00 & 6,00 & 15,00 & $11,11 \%$ & $13,04 \%$ & 236 & $11,11 \%$ \\
\hline SD & 46,21 & 44,05 & 44,96 & 6,55 & 5,00 & 8,13 & $0,00 \%$ & $0,50 \%$ & 47 & $1,25 \%$ \\
\hline
\end{tabular}

Table 1: $3 D$ ultrasound-estimated volume measurements for technicians $A$ and $B$, true volume measurements (C) and measurement deviations between A, B and C. Volume measurements for technician $B$ are lacking in phantoms number 13 and 25 due to balloon puncture. 


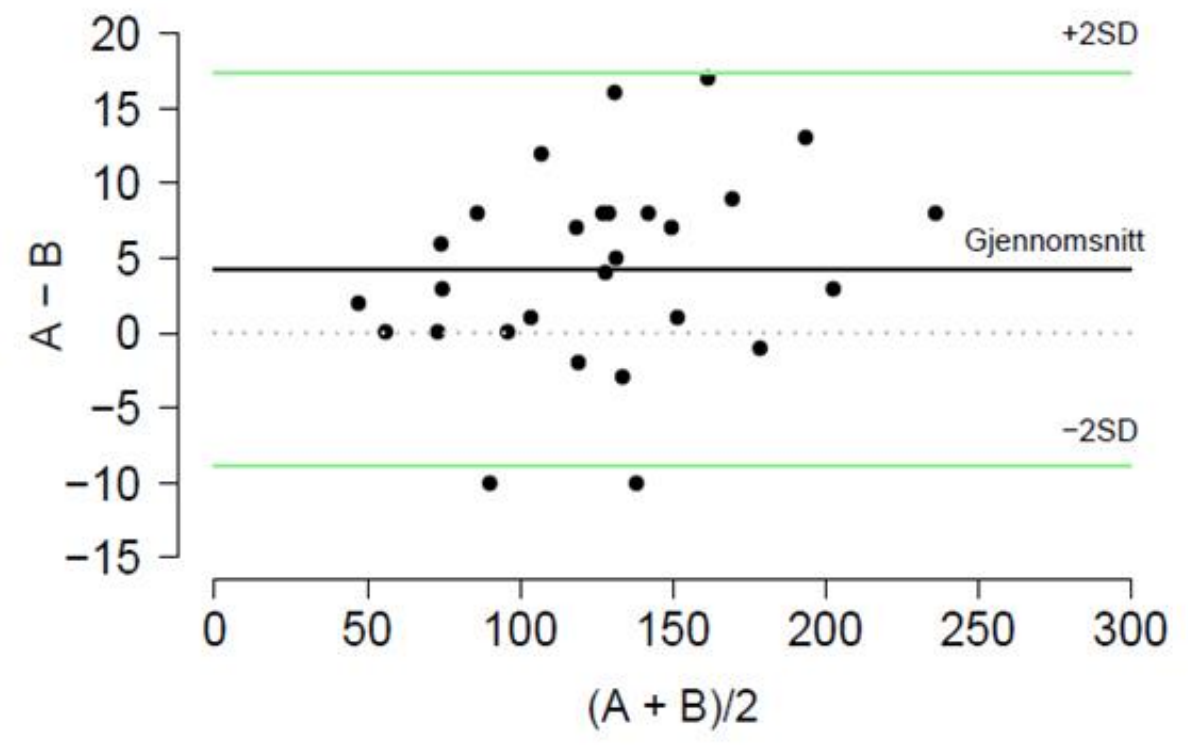

Figure 7: Limits of agreement between technicians A and B. Bland-Altman plot.

\section{Discussion}

Three-dimensional ultrasound of our phantom AAA model yielded excellent correlation between estimated and true volumes. The ultrasound procedure was quick and easy to perform, and accuracy and precision were very good. We did not encounter any difficulties when using 3D US to image sizes and shapes similar to those of AAAs in this proof-of-concept study.

A generally recognized limitation of ultrasound imaging is operator dependency. In our study, the inter-rater agreement between the two technicians was very good. However, if multiple technicians had performed the ultrasound scans, the results might have been closer to normal distribution.

The in vitro experiment that we performed in the present study validated our 3D US volume measurement technique. The major advantages of such a controlled experiment are that there are few variables, it is easily controlled, and the results are reliable. However, our in vitro results cannot be considered directly relevant to clinical settings, and clinical investigations are required for a variety of reasons: The phantom used in this experiment was filled with water, which interacts differently with ultrasound waves compared to tissue. While ultrasound waves travel through water with very little attenuation and scattering, in the body, ultrasound images of AAA will be affected by respiration, cardiac, and intestinal movements. In addition, ultrasound images may be affected by several artifacts due to attenuation and scattering through the different types of tissue. 
Three-dimensional Ultrasound Volume Measurements in an Aortic Aneurysm Model

A number of studies that utilized different 3D US methods and types of equipment have yielded good results for volume measurement of kidneys and fetal lungs and bladder ${ }^{8-10}$. In particular, previous studies that used VOCAL ${ }^{\mathrm{TM}}$ ultrasound software found good precision and accuracy for organ volume measurements ${ }^{9,13}$. There are many types of commercially available 3D US systems with which to measure volume, each with its own limitations, advantages, and disadvantages. However, we were unable to identify any previous studies validating the use of a commercially available 3D US probe and its accompanying software to measure AAA volume.

It is important to monitor the AAA sac after surgery to detect increases in volume, since ELs that increase the size of the aneurysmal sac require additional treatment ${ }^{5,6}$. A previous study of follow-up after EVAR found that 3D diameter measurements are more precise than 2D measurements of diameter ${ }^{4}$. Recently, clinical and experimental studies have yielded promising results for 3D US compared to CTA measurements of the volume of AAAs using modified research 3D probe and software systems ${ }^{14,15}$. There is a rapid development in this field. The use of matrix probes may improve volume recordings, thus reducing the risk of errors due to bowel and respiratory movement. This study validated a commercially available product that is fast and easy to learn. We are currently planning a clinical trial in which we will use the 3D US volume measurement technique described herein for follow-up examinations after EVAR surgery to treat AAA.

\section{Conclusion}

The GE 3D/4D RAB 2-5D curved linear electromechanical wideband array transducer and the VOCAL $^{\mathrm{TM}}$ software program were well suited for performing $3 \mathrm{D}$ volume measurements in our phantom AAA model. Inter-rater agreement was very good, and the method was fast and easy to perform. These results must be confirmed in clinical studies. 
Johnsen, Jonung, Laxdal, Pedersen

Three-dimensional Ultrasound Volume Measurements in an Aortic Aneurysm Model

\section{References}

1. Dalman RL, Mell M. Management of Asymptomatic Abdominal Aortic Aneurysms. Wolters Kluver, 2018. Official reprint from UpToDateUp www.uptodate.com/contents/management-of-asymptomatic-abdominal-aortic-aneurysm

2. Chaer RA. Endovascular repair of abdominal aortic aneurysm. Wolters Kluver, 2018. Official reprint from UpToDate www.uptodate.com/contents/endovascular-repair-of-abdominal-aortic-aneurysm

3. Patel R, Sweeting MJ, Powell JT, Greenhalgh RM; EVAR trial investigators. Endovascular versus open repair of abdominal aortic aneurysm in 15-years' follow-up of the UK endovascular aneurysm repair trial 1 (EVAR trial 1): A randomised controlled trial. Lancet. 2016 Nov 12;388(10058):2366-2374. DOI/10.1016/S0140-6736(16)31135-7

4. Mirza TA, Karthikesalingam A, Jackson D, et al. Duplex ultrasound and contrastenhanced ultrasound versus computed tomography for the detection of endoleak after EVAR: systematic review and metaanalysis. European Journal of Vascular and Endovascular Surgery2010;39:418-28.

DOI/10.1016/j.ejvs.2010.01.001

5. Silverberg D, Baril DT, Ellozy SH, et al. An 8-year experience with type II endoleaks: natural history suggests selective intervention is a safe approach. Journal of Vascular Surgery 2006 Sep; 44 (3): 453-9 DOI/10.1016/j.jvs.2006.04.058

6. Brown A, Saggu GK, Bown MJ, Sayers RD, Sidloff DA. Type II endoleaks: Challenges and solutions. Vascular Health and Risk Management 2016;12:53-63 DOI/ 10.2147/VHRM.S81275. eCollection 2016

7. Bargellini I, Cioni R, Petruzzi P, et al. Endovascular Repair of Abdominal Aortic Aneurysms: Analysis of Aneurysm Volumetric Changes at Med-Term Follow-Up. Cardiovascular Interventional Radiology: 2005;28:426-32. DOI/10.1007/s00270-004-0171-9

8. Gilja $\mathrm{OH}$, Smievoll Al, Thune $\mathrm{N}$, et al. In vivo comparison of $3 \mathrm{~d}$ ultrasonography and magnetic resonance imaging in volume estimation of human kidneys. Ultrasound in Medicine and Biology, Vol 21, No 1, pp.25-32, 1995.

DOI/10.1016/0301-5629(94)00082-4 
Johnsen, Jonung, Laxdal, Pedersen

Three-dimensional Ultrasound Volume Measurements in an Aortic Aneurysm Model

9. Ruano R. Martinovic J, Dommergues M, Aubry MC, Dumez Y, Banchi A. Accuracy of fetal lung volume assessed by three-dimensional sonography. Ultrasound in Obstetrics \& Gynecology. 2005;26: 725-30. DOI/10.1002/uog.2624

10. Peixoto-Filho FM, Sà RA, Lopes LM et al. Three-dimensional ultrasound fetal urinary bladder volume measurement: reliability of rotational (VOCAL) techinque using different steps of rotation. Archives of Gynecology and Obstetrics . 2007;276:345-9. DOI/10.1007/s00404-007-0360-2

11. Strømmen K, Stormark TA, Iversen BM, Matre K. Volume estimation of small phantoms and rat kidneys using three-dimensional ultrasonography and a position sensor. Ultrasound in Medicine and Biology 2004;30:1109-17. DOI/10.1016/j.ultrasmedbio.2004.08.005

12. Kot BCW, Sin DMH, Ying M. Evaluation of the Accuracy and Reliability of Two 3Dimensional Sonography Methods in Volume Measurement of Small Structures: An In Vitro Phantom Study. Journal of Clinical Ultrasound 2009, vol.37, no.2. DOI /10.1002/jcu.20525

13. Raine-Fennig NJ, Clewes JS, Kendall NR et al. The inter-observer reliability and validity of volume calculation from three-dimensional ultrasound datasets in the in vitro setting. Ultrasound in Obstetrics \& Gynecology 2003;21:283-91. DOI/10.1002/uog.61

14. Arsicot $M$, Lathelize $H$, Martinez $R$ et al. Follow-up of aortic stent grafts: comparison of the volumetric analysis of the aneurysm sac by ultrasound and CT. Annals of Vascular Surgery 2014;28:1618-28. DOI/10.1016/j.avsg.2014.03.034

15. Raine-Fennig NJ, Clewes JS, Kendall NR et al. The interobserver reliability and validity of volume calculation from three-dimensional ultrasound datasets in the in vitro setting. Ultrasound in Obstetrics \& Gynecology. 2003;21:283-91. DOI/10.1002/uog.61 